ISSN 1420-3049

www.mdpi.com/journal/molecules

Communication

\title{
Synthesis and Antimicrobial Evaluation of Some New Oxadiazoles Derived from Phenylpropionohydrazides
}

Neeraj Kumar Fuloria ${ }^{1, *}$, Vijender Singh ${ }^{1}$, Mohammad Shaharyar ${ }^{2}$ and Mohammad Ali ${ }^{3}$

1 Department of Pharmacy, Rameesh Institute of Vocational and Technical Education, 3-Knowledge Park-1, Kasna road, Greater NOIDA, India; E-mail: mahalwal_vs@yahoo.com (V.S.)

2 Department of Pharmaceutical Chemistry, Faculty of Pharmacy, Jamia Hamdard, Hamdard Nagar, New Delhi-110062, India; E-mail: yarmsy@rediffmail.com (M.S.)

3 Department of Pharmacognosy \& Phytochemistry, Faculty of Pharmacy, Jamia Hamdard, Hamdard Nagar, New Delhi-110062, India

* Author to whom correspondence should be addressed; E-mail: nfuloria@rediffmail.com; Tel.: 01122175107

Received: 14 April 2009; in revised form: 12 May 2009 / Accepted: 13 May 2009 /

Published: 20 May 2009

\begin{abstract}
In this study a series of new 1-(2-aryl-5-phenethyl-1,3,4-oxadiazol-3(2H)yl)ethanones 2a-e was synthesized by the cyclization of imines 1a-e using acetic anhydride. The products were evaluated for anti-bacterial and anti-fungal activity. Among the newly synthesized compounds, 1-(2-(4-(dimethylamino)phenyl)-5-phenethyl-1,3,4oxadiazol-3(2H)-yl)ethanone (2a) and 1-(2-(4-chlorophenyl)-5-phenethyl-1,3,4-oxadiazol$3(2 \mathrm{H})-y l) e t h a n o n e(\mathbf{2 b})$ were found to possess maximum activity against the tested strains of $S$. aureus and P. aeruginosa. It was concluded that para-substitution enhances the activity of synthesized oxadiazoles.
\end{abstract}

Keywords: phenylpropionohydrazide; imines; oxadiazoles; antibacterial; antifungal

\section{Introduction}

It is an established fact that oxadiazoles, imines and propanoates exhibits antimitotic [1], antikinetoplastid [2], antitussive [3], hybrid COX-2 inhibitor/nitric oxide donor [4], antimycotic [5], anti-inflammatory [6], analgesic [7], antimicrobial and anticonvulsant [8-12] activities. Moreover 
esters and hydrazides can be converted into imines, which are precursor for oxadiazoles [8-12]. The literature has reported different biological activities and method of synthesis for oxadiazoles [1-12]. Hence an attempt was made to convert some $N$-(substituted benzylidene)-3-phenylpropionohydrazides into novel 1-(2-aryl-5-phenethyl-1,3,4-oxadiazol-3(2H)-yl)ethanones. The novel compounds were characterized and further investigated for anti-bacterial and anti-fungal activities.

\section{Results and Discussion}

\subsection{Chemistry}

The treatment of $N$-(substituted benzylidene)-3-phenylpropionohydrazides 1a-e, with acetic anhydride yielded 1-(2-aryl-5-phenethyl-1,3,4-oxadiazol-3(2H)-yl)ethanones 2a-e (Scheme 1).

Scheme 1. Synthesis of Oxadiazoles 2a-e.

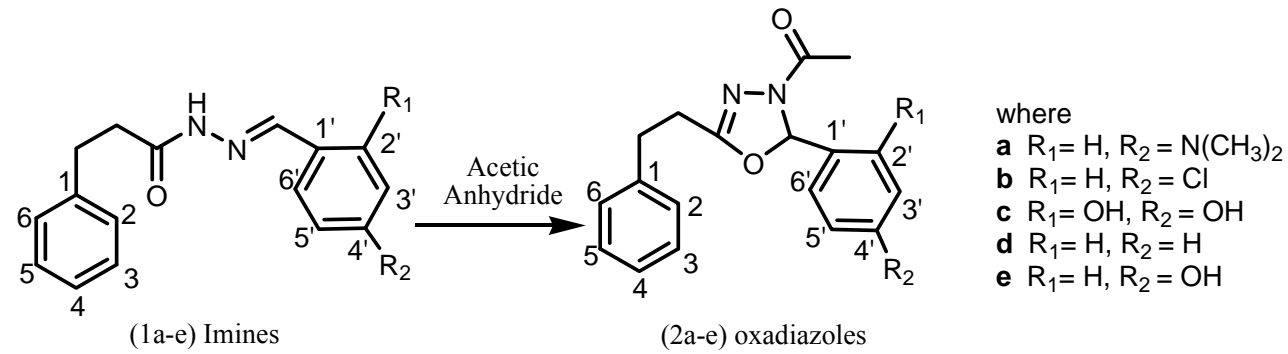

The carbonylamino and imino groups in compounds 1a-e, were found to cyclize to form oxadiazole rings when reacted with acetic anhydride. The assigned structures, molecular formulae and the anomeric configuration of the newly synthesized oxadiazoles 2a-e were further confirmed and supported by mass, ${ }^{1} \mathrm{H}-\mathrm{NMR}$ and IR spectrometry. The fragmentation pattern of compound 2a due to absence of $\mathrm{C}_{8} \mathrm{H}_{9}, \mathrm{C}_{12} \mathrm{H}_{13} \mathrm{~N}_{2} \mathrm{O}_{2}, \mathrm{C}_{16} \mathrm{H}_{19} \mathrm{~N}, \mathrm{C}_{12} \mathrm{H}_{14} \mathrm{~N}_{3} \mathrm{O}_{2}, \mathrm{C}_{13} \mathrm{H}_{16} \mathrm{~N}_{3} \mathrm{O}_{2}, \mathrm{C}_{15} \mathrm{H}_{18} \mathrm{~N}_{3} \mathrm{O}_{2}$ groups given in Figure 1 , as an example further supported in identification of molecular structures of compounds 2a-e.

Figure 1. fragmentation pattern of oxadiazole 2a.

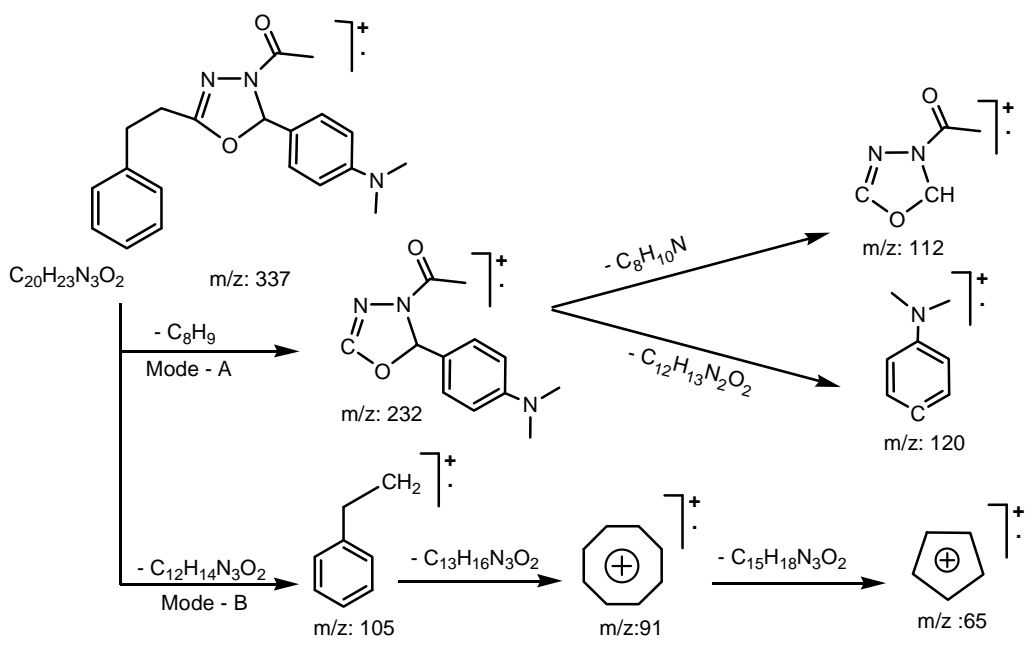

The absence of specific group fragments in the mass spectra of compounds $2 \mathbf{b}-\mathbf{e}\left(\mathrm{C}_{8} \mathrm{H}_{9}\right.$, $\mathrm{C}_{12} \mathrm{H}_{13} \mathrm{~N}_{2} \mathrm{O}_{2}, \mathrm{C}_{14} \mathrm{H}_{13} \mathrm{Cl}, \mathrm{C}_{10} \mathrm{H}_{8} \mathrm{ClN}_{2} \mathrm{O}_{2}, \mathrm{C}_{11} \mathrm{H}_{10} \mathrm{ClN}_{2} \mathrm{O}_{2}, \mathrm{C}_{13} \mathrm{H}_{12} \mathrm{ClN}_{2} \mathrm{O}_{2}$ in the case of 2b; $\mathrm{C}_{8} \mathrm{H}_{9}$, 
$\mathrm{C}_{12} \mathrm{H}_{13} \mathrm{~N}_{2} \mathrm{O}_{2},-\mathrm{C}_{14} \mathrm{H}_{14} \mathrm{O}_{2}, \mathrm{C}_{10} \mathrm{H}_{9} \mathrm{~N}_{2} \mathrm{O}_{4}, \mathrm{C}_{11} \mathrm{H}_{11} \mathrm{~N}_{2} \mathrm{O}_{4}, \mathrm{C}_{13} \mathrm{H}_{13} \mathrm{~N}_{2} \mathrm{O}_{4}$ in 2c; $\mathrm{C}_{8} \mathrm{H}_{9}, \mathrm{C}_{12} \mathrm{H}_{13} \mathrm{~N}_{2} \mathrm{O}_{2}, \mathrm{C}_{14} \mathrm{H}_{14}$, $\mathrm{C}_{10} \mathrm{H}_{9} \mathrm{~N}_{2} \mathrm{O}_{2}, \mathrm{C}_{11} \mathrm{H}_{11} \mathrm{~N}_{2} \mathrm{O}_{2}, \mathrm{C}_{13} \mathrm{H}_{13} \mathrm{~N}_{2} \mathrm{O}_{2}$ 2d; and $\mathrm{C}_{8} \mathrm{H}_{9}, \mathrm{C}_{12} \mathrm{H}_{13} \mathrm{~N}_{2} \mathrm{O}_{2}, \mathrm{C}_{14} \mathrm{H}_{14} \mathrm{O}, \mathrm{C}_{10} \mathrm{H}_{9} \mathrm{~N}_{2} \mathrm{O}_{3}, \mathrm{C}_{11} \mathrm{H}_{11} \mathrm{~N}_{2} \mathrm{O}_{3}$, $\mathrm{C}_{13} \mathrm{H}_{13} \mathrm{~N}_{2} \mathrm{O}_{3}$ in 2e) was a key to establish their molecular structures. The purity of the compounds was checked by melting point, TLC and elemental analysis results, which were within $\pm 0.4 \%$ of the theoretical values.

\subsection{Biological activity}

The newly synthesized compounds 2a-e were screened for antibacterial activity against freshly cultured strains of $S$. aureus (SA) and P. aeruginosa (PA) using sterile nutrient agar media and for antifungal activity against freshly cultured strains of $C$. albicans (CA) and A. flavus (AF) using sterile sabouraud's agar medium by the disk diffusion method at a concentration of $2 \mathrm{mg}$ per $\mathrm{mL}$. using DMF as solvent. The results were recorded in duplicate using ampicillin and fluconazole at a concentration of $1 \mathrm{mg}$ per $\mathrm{mL}$ as standards.

Table 1. Antimicrobial activity-sensitivity testing of 2a-e.

\begin{tabular}{ccc|cc}
\hline \multirow{2}{*}{$\begin{array}{c}\text { Compound } \\
\text { No. }\end{array}$} & \multicolumn{4}{c}{ Zone of inhibition in mm } \\
\cline { 2 - 5 } & Antibacterial Activity & \multicolumn{2}{c}{ Antifungal Activity } \\
\cline { 2 - 5 } & SA & PA & CA & AF \\
\hline 2a & 24 & 24 & 16 & 15 \\
2b & 25 & 24 & 15 & 13 \\
2c & 23 & 20 & 13 & 12 \\
2d & 22 & 23 & 16 & 13 \\
2e & 19 & 20 & 16 & 15 \\
\hline Ampicillin & 25 & 24 & - & - \\
\hline Fluconazole & - & - & 17 & 16 \\
\hline
\end{tabular}

Among newly synthesized derivatives, compounds $\mathbf{2 a}$ and $\mathbf{2} \mathbf{b}$ were found to be equipotent to ampicillin when tested against the strains of $S$. aureus, and $P$. aeruginosa, whereas some of the newly synthesized compounds like 2a, 2d and $\mathbf{2 e}$ were found to possess good antibacterial and antifungal activity when tested against $S$. aureus, P. aeruginosa, C. albicans and A. flavus (Table 1).

\section{Experimental Section}

\subsection{General}

Melting points of newly synthesized compounds were determined using Thomas Hoover apparatus. IR spectra were recorded (in KBr) on a Bruker PCIR, ${ }^{1} \mathrm{H}-\mathrm{NMR}$ on Bruker, DPX 300 and mass spectra on MASPEC (MSW/9629). Purity of synthesized compounds was checked by TLC aluminium sheets - silica gel 60 F254 (0.2 mm). 
3.2. General procedure for the synthesis of 1-(2-aryl-5-phenethyl-1,3,4-oxadiazol3(2H)-yl)ethanones (2a-e)

A mixture of compound 1a-e $(0.01 \mathrm{~mol})$ derived from 3-phenyl propane hydrazide was refluxed with acetic anhydride $(0.01 \mathrm{~mol})$ for 12 hours in the presence of zinc chloride. The product formed was isolated by filtration and recrystallized from methanol to yield compounds 2a-e.

1-(2-(4-(Dimethylamino)phenyl)-5-phenethyl-1,3,4-oxadiazol-3(2H)-yl)ethanone (2a): Pale yellow crystals; Yield 65.8\%; mp 225-226 ${ }^{\circ} \mathrm{C} ;{ }^{1} \mathrm{H}-\mathrm{NMR} \delta(\mathrm{ppm}): 2.04\left(3 \mathrm{H}, \mathrm{s},-\mathrm{CO}-\mathrm{CH}_{3}\right), 2.32(2 \mathrm{H}, \mathrm{t}, 6.9 \mathrm{~Hz}$, - $\left.\mathrm{CH}_{2}-\mathrm{C}-\mathrm{O}-\right), 2.65$ (2H, t, 6.9Hz, Ar- $\left.\mathrm{CH}_{2}\right), 2.89\left(6 \mathrm{H}, \mathrm{s},-\mathrm{N}\left(\mathrm{CH}_{3}\right)_{2}\right), 6.52\left(2 \mathrm{H}, \mathrm{d}, 8.1 \mathrm{~Hz}, \mathrm{Ar}-\mathrm{H} 3^{\prime}\right.$ \& 5'), $6.65\left(1 \mathrm{H}, \mathrm{s},-\mathrm{N}-\mathrm{CH}-\mathrm{Ar}^{\prime}\right), 7.01\left(2 \mathrm{H}, \mathrm{d}, 8.2 \mathrm{~Hz}, \mathrm{Ar}^{\prime}-\mathrm{H} 2^{\prime} \& 6^{\prime}\right), 7.18-7.31$ (5H, m, Ar-H2, 3, 4, 5 \& 6); FTIR: $2924\left(\mathrm{C}-\mathrm{H}\right.$ of $\left.\mathrm{CH}_{2}\right), 1688(\mathrm{C}=\mathrm{O}), 1611(\mathrm{C}=\mathrm{N}), 1259(\mathrm{C}-\mathrm{O}-\mathrm{C}) \mathrm{cm}^{-1}$; Anal. Calcd. for $\mathrm{C}_{20} \mathrm{H}_{23} \mathrm{~N}_{3} \mathrm{O}_{2}$ (337.42): C: 71.19, H: 6.87, N: 12.45. found: $\mathrm{C}: 71.16, \mathrm{H}: 6.85, \mathrm{~N}: 12.43 ; \mathrm{MS}: \mathrm{m} / \mathrm{z}: 337\left(\mathrm{M}^{+}\right)$, 232 (base peak), 120, 112, 105, 91, 65 .

1-(2-(4-Chlorophenyl)-5-phenethyl-1,3,4-oxadiazol-3(2H)-yl)ethanone (2b): White crystals; Yield 64.9\%; mp 212-213 ${ }^{\circ} \mathrm{C}$; ${ }^{1} \mathrm{H}-\mathrm{NMR} \delta$ (ppm): 2.11 (3H, s, -CO-CH $\left.\mathrm{CH}_{3}\right), 2.38$ (2H, t, 6.5Hz, -CH $\left.-\mathrm{C}-\mathrm{O}-\right)$, $2.72\left(2 \mathrm{H}, \mathrm{t}, 6.6 \mathrm{~Hz}, \mathrm{Ar}-\mathrm{CH}_{2}\right), 6.64\left(1 \mathrm{H}, \mathrm{s},-\mathrm{N}-\mathrm{CH}-\mathrm{Ar}^{\prime}\right), 7.14\left(2 \mathrm{H}, \mathrm{d}, 8.3 \mathrm{~Hz}, \mathrm{Ar}^{\prime}-\mathrm{H} 2^{\prime} \& 6^{\prime}\right), 7.20(2 \mathrm{H}, \mathrm{d}$, 8.1Hz, Ar'-H3' \& 5'), 7.24-7.37 (5H, m, Ar-H2, 3, 4, 5 \& 6); FT-IR: 1608 (C=N), 2928 (C-H of CH$)_{2}$, $1681(\mathrm{C}=\mathrm{O}), 1256(\mathrm{C}-\mathrm{O}-\mathrm{C}) \mathrm{cm}^{-1}$; Anal. Calcd. for $\mathrm{C}_{18} \mathrm{H}_{17} \mathrm{~N}_{2} \mathrm{O}_{2} \mathrm{Cl}$ (328.79): C: 65.75, H: 5.21, N: 8.52. Found: C: 65.72, H: 5.20, N: 8.50; MS: m/z $328\left(\mathrm{M}^{+}\right), 223$ (base peak), 112, 111, 105, 91, 65.

1-(2-(2,4-Dihydroxyphenyl)-5-phenethyl-1,3,4-oxadiazol-3(2H)-yl) ethanone (2c): Yellow brown crystals; Yield 59.2\%; mp 219-220 ${ }^{\circ} \mathrm{C}$; ${ }^{1} \mathrm{H}-\mathrm{NMR} \delta(\mathrm{ppm}): 2.07\left(3 \mathrm{H}, \mathrm{s},-\mathrm{CO}-\mathrm{CH}_{3}\right), 2.32(2 \mathrm{H}, \mathrm{t}$, $\left.6.8 \mathrm{~Hz},-\mathrm{CH}_{2}-\mathrm{C}-\mathrm{O}-\right), 2.62\left(2 \mathrm{H}, \mathrm{t}, 6.8 \mathrm{~Hz}, \mathrm{Ar}_{-} \mathrm{CH}_{2}\right), 5.22(1 \mathrm{H}, \mathrm{s}, 4-\mathrm{OH}), 5.28(1 \mathrm{H}, \mathrm{s}, 2-\mathrm{OH}), 6.14(1 \mathrm{H}, \mathrm{d}$, $\left.2.6 \mathrm{~Hz}, \mathrm{Ar}^{\prime}-\mathrm{H3}^{\prime}\right), 6.28\left(1 \mathrm{H}, \mathrm{dd}, 2.8,7.6 \mathrm{~Hz}, \mathrm{Ar}^{\prime}-\mathrm{H} 5^{\prime}\right), 6.60\left(1 \mathrm{H}, \mathrm{s},-\mathrm{N}-\mathrm{CH}-\mathrm{Ar}^{\prime}\right), 6.88\left(1 \mathrm{H}, \mathrm{d}, 7.9 \mathrm{~Hz}, \mathrm{Ar}^{\prime}-\right.$ H6'), 7.19-7.32 (5H, m, Ar-H2, 3, 4, 5 \& 6 ); FT-IR: $3516(\mathrm{OH}), 2926\left(\mathrm{C}-\mathrm{H}\right.$ of $\left.\mathrm{CH}_{2}\right), 1683(\mathrm{C}=\mathrm{O})$, $1617(\mathrm{C}=\mathrm{N}), 1253$ (C-O-C) $\mathrm{cm}^{-1}$; Anal. Calcd. for $\mathrm{C}_{18} \mathrm{H}_{18} \mathrm{~N}_{2} \mathrm{O}_{4}$ (326.34): C: 66.25, H: 5.56, N: 8.58. Found: $\mathrm{C}: 66.22, \mathrm{H}: 5.52, \mathrm{~N}: 5.54$; MS: m/z $326\left(\mathrm{M}^{+}\right), 221$ (base peak), 112, 109, 105, 91, 65.

1-(5-Phenethyl-2-phenyl-1,3,4-oxadiazol-3(2H)-yl)ethanone (2d): White crystals; Yield 60.3\%; mp 203-204 ${ }^{\circ} \mathrm{C} ;{ }^{1} \mathrm{H}-\mathrm{NMR} \delta$ (ppm): $2.02\left(3 \mathrm{H}, \mathrm{s},-\mathrm{CO}-\mathrm{CH}_{3}\right), 2.30\left(2 \mathrm{H}, \mathrm{t}, 6.4 \mathrm{~Hz},-\mathrm{CH}_{2}-\mathrm{C}-\mathrm{O}-\right), 2.61(2 \mathrm{H}, \mathrm{t}$, 6.5Hz, Ar- $\left.\mathrm{CH}_{2}\right), 6.61\left(1 \mathrm{H}, \mathrm{s},-\mathrm{N}-\mathrm{CH}-\mathrm{Ar}^{\prime}\right), 7.15-7.29\left(10 \mathrm{H}, \mathrm{m}, \mathrm{Ar}^{\prime}-\mathrm{H} 2^{\prime}, 3^{\prime}, 4^{\prime}, 5^{\prime}\right.$ \& $6^{\prime}$ \& $\mathrm{Ar}-\mathrm{H} 2,3,4,5$ \& 6); FT-IR: $1610(\mathrm{C}=\mathrm{N}), 2925\left(\mathrm{C}-\mathrm{H}\right.$ of $\left.\mathrm{CH}_{2}\right), 1686(\mathrm{C}=\mathrm{O}), 1249(\mathrm{C}-\mathrm{O}-\mathrm{C}) \mathrm{cm}^{-1}$; Anal. Calcd. for $\mathrm{C}_{18} \mathrm{H}_{18} \mathrm{~N}_{2} \mathrm{O}_{2}$ (294.35): $\mathrm{C}: 73.45, \mathrm{H}: 6.16, \mathrm{~N}: 9.52$. Found: $\mathrm{C}: 73.42, \mathrm{H}: 6.14, \mathrm{~N}: 9.50 ; \mathrm{MS}: \mathrm{m} / \mathrm{z}$ $294\left(\mathrm{M}^{+}\right), 189$ (base peak), 105, 91, 77, 65.

1-(2-(4-Hydroxyphenyl)-5-phenethyl-1,3,4-oxadiazol-3(2H)-yl)ethanone (2e): Orange crystals; Yield 62.4\%; mp 213-214 ${ }^{\circ} \mathrm{C}$; ${ }^{1} \mathrm{H}-\mathrm{NMR} \delta$ (ppm): 2.05 (3H, s, -CO-CH $\mathrm{CH}_{3}, 2.35$ (2H, t, 6.6Hz, -CH $\left.-\mathrm{C}-\mathrm{O}-\right)$, $2.67\left(2 \mathrm{H}, \mathrm{t}, 6.5 \mathrm{~Hz}, \mathrm{Ar}-\mathrm{CH}_{2}\right), 5.26(1 \mathrm{H}, \mathrm{s}, 4-\mathrm{OH}), 6.61\left(1 \mathrm{H}, \mathrm{s},-\mathrm{N}-\mathrm{CH}-\mathrm{Ar}^{\prime}\right), 6.68\left(2 \mathrm{H}, \mathrm{d}, 7.8 \mathrm{~Hz}, \mathrm{Ar}^{\prime}-\mathrm{H}^{\prime}\right.$ \& 5'), $7.03\left(2 \mathrm{H}, \mathrm{d}, 7.5 \mathrm{~Hz}, \mathrm{Ar}^{\prime}-\mathrm{H} 2^{\prime} \&\right.$ 6'), 7.16-7.29 (5H, m, Ar-H2, 3, 4, 5 \& 6); FT-IR: $3512(\mathrm{OH})$, $2920\left(\mathrm{C}-\mathrm{H}\right.$ of $\left.\mathrm{CH}_{2}\right), 1680(\mathrm{C}=\mathrm{O}), 1613(\mathrm{C}=\mathrm{N}), 1249(\mathrm{C}-\mathrm{O}-\mathrm{C}) \mathrm{cm}^{-1}$; Anal. Calcd. for $\mathrm{C}_{18} \mathrm{H}_{18} \mathrm{~N}_{2} \mathrm{O}_{3}$ 
(310.34): C: 69.66, H: 5.85, N: 9.03. Found: C: 69.64, H: 5.82, N: 9.01; MS: m/z $310\left(\mathrm{M}^{+}\right), 205$ (base peak), 112, 105, 93, 91, 65 .

\section{Conclusions}

Both analytical and spectral data (IR, ${ }^{1} \mathrm{H}-\mathrm{NMR}, \mathrm{MS}$ ) of all the synthesized compounds were in full agreement with the proposed structure. After comparing the antimicrobial results of compounds 2a-e, it was concluded that the incorporation of an oxadiazole moiety in phenylpropionyl derivatives enhances their antimicrobial activity and also para-substitution in the $\mathrm{Ar}^{\prime}$ group of the oxadiazoles was found to enhance their potency, especially in compound $\mathbf{2 a}$ and $\mathbf{2 b}$. Further studies to acquire more information about structure activity relationship are in progress in our laboratory.

\section{Acknowledgements}

The authors are thankful to CDRI, Lucknow, IIT Delhi and IIT Chennai for carrying out spectral studies. Thanks are also due to Rameesh Institute of Vocational and Technical Education, Greater Noida, for providing necessary facilities.

\section{References}

1. Rai, K.M.; Linganna, N.O. Synthesis and evaluation of antimitotic activity of alkylated 2-amino1,3,4-oxadiazole derivatives. Farmaco 2000, 55, 389-392.

2. Cottrell, D.M.; Capers, J.; Salem, M.M.; Fradley, K.D.; Croft, S.L.; Werbovetz, K.A. Antikinetoplastid activity of 3-aryl-5-thiocyanatomethyl-1,2,4-oxadiazoles. Bioorg. Med. Chem. 2004, 12, 2815-2824.

3. Harsányi, K.; Kiss, P.; Korbonits, D.; Malyáta, I.R. The synthesis of an antitussive action derivative of 1,2,4-oxadiazole, 3-(2,2-diphenylethyl)-5-(2-piperidinoethyl)-1,2,4-oxadiazole. Arzn. Forsch. 1966, 16, 615-617.

4. Carlos, V.; Rao, P.P.N.; Robert, M.; Edward, K.E. Synthesis and biological evaluation of 3,4diphenyl-1,2,5-oxadiazole-2-oxides and 3,4-diphenyl-1,2,5-oxadiazoles as potential hybrid COX2 inhibitor/nitric oxide donor agents. Bioorg. Med. Chem. 2005, 13, 2749-2757.

5. Mazzone, G.; Bonina, F. Synthesis and antimycotic activity of 3-methylamino derivatives of various 2-mercapto-5-aryl-1,3,4-oxadiazoles. Farmaco 1979, 34, 390-402.

6. Balsamo, A.; Bertini, S.; Gervasi, G.; Lapucci, A.; Nencetti, S.; Orlandini, E.; Rapposelli, S.; Rossello, A.; Soldani, G. Enantiopure 3-(arylmethylidene)aminoxy-2-methylpropionic acids: synthesis and antiinflammatory properties. Eur. J. Med. Chem. 2001, 36, 799-807.

7. Kido, H.; Murakami, N.; Ito, A.; Kimura, K.; Kodera, N.; Doi, T.; Naruse, T. Anti-inflammatory, analgesic and anti-pyretic effects of $d$-2-[4-(3-methyl-2-thienyl)phenyl]propionic acid (m-5011), a new non-steroidal anti-inflammatory drug, in rats and guinea pigs. Jpn. J. Pharmaco. 1998, 76, $75-86$.

8. Fuloria, N.K.; Singh, V.; Shaharyar, M.; Ali, M. Synthesis, characterization and biological studies of novel imines and azetidinones derivatives of haloaryloxy moiety. Asian J. Chem. 2008, 20, 6457-6462. 
9. Fuloria, N.K.; Singh, V.; Shaharyar, M.; Ali, M. Synthesis, characterization and biological studies of new schiff bases and azetidinones derived from propionic acid derivatives. Asian J. Chem. 2008, 20, 4891-4900.

10. Fuloria, N.K.; Singh, V.; Shaharyar, M.; Ali, M. Antimicrobial evaluation of imines and thiazolidinones derived from 3-phenyl propanehydrazide. Acta. Pol. Pharm. Drug. Res. 2009, 66, 141-146.

11. Nassar, O.M. Synthesis of certain 1,3,4-oxadiazole derivatives as potential anticonvulsant. Ind. J. Heterocycl. Chem. 1997, 7,105-108.

12. Fuloria, N.K.; Singh, V.; Shaharyar, M.; Ali, M. Synthesis and antimicrobial studies of novel imines and oxadiazoles. South Braz. J. Chem. 2008, 16, 11-22.

Sample Availability: Samples of the compounds 2a-e are available from the authors.

(C) 2009 by the authors; licensee Molecular Diversity Preservation International, Basel, Switzerland. This article is an open-access article distributed under the terms and conditions of the Creative Commons Attribution license (http://creativecommons.org/licenses/by/3.0/) 